\title{
Manipulation of mobile spin coherence using magnetic-field-free electron spin resonance
}

\author{
H. Sanada ${ }^{1 \star}$, Y. Kunihashi' ${ }^{1}$ H. Gotoh ${ }^{1}$, K. Onomitsu' ${ }^{1}$, M. Kohda ${ }^{2}$, J. Nitta ${ }^{2}$, P. V. Santos ${ }^{3}$ \\ and T. Sogawa ${ }^{1}$
}

Electron spin resonance (ESR) has applications in the manipulation of individual electron spins for quantum information processing ${ }^{1-6}$. In general, ESR requires two external magnetic fields: a static field $\left(B_{0}\right)$ to split the spin states in energy and an oscillating field $\left(B_{1}\right)$ with the frequency resonant to the splitting energy. However, spin manipulation methods relying on real magnetic fields-much broader than the size of individual electrons-are energetically inefficient and unsuitable for future device applications. Here we demonstrate an alternative approach where the spin-orbit interaction ${ }^{7}$ of trajectory-controlled electrons induces effective $B_{0}$ and $B_{1}$ fields. These fields are created when electron spins surf on sound waves ${ }^{8-10}$ along winding semiconductor channels. The resultant spin dynamics-mobile spin resonance-is equivalent to the usual ESR but requires neither static nor time-dependent real magnetic fields to manipulate electron spin coherence.

In the electron systems in inhomogeneous magnetic fields or systems with spin-orbit coupling, the motion of electrons is converted to time-dependent effective magnetic fields. The latest studies have reported that ESR can be driven by effective $\mathbf{B}_{1}$ fields, which are produced by the reciprocating motion of electrons with spatially dependent spin splitting ${ }^{11-13}$ or with spin-orbit coupled systems ${ }^{14-16}$, but these techniques still need external $\mathbf{B}_{0}$ fields. A promising approach for incorporating effective $\mathbf{B}_{0}$ fields is the use of long-distance spin transport, which induces spin precessions around the static spin-orbit magnetic fields $s^{9,17}$. In addition, spin-qubit operations using moving quantum dots have been theoretically proposed ${ }^{18}$. Thus, we expect all of the magnetic fields needed for ESR to be replaced with spin-orbit effective magnetic fields $\left(\mathbf{B}^{\mathrm{SO}}\right)$ by using trajectorycontrolled travelling electrons.

Mobile spin resonance is based on the dependence of $\mathbf{B}^{\mathrm{SO}}$ on the electron momentum vector $\mathbf{k}$. For simplicity, we assume a two-dimensional electron system in a (001) III-V quantum well with only $k$-linear terms in the Dresselhaus spin-orbit interaction ${ }^{19}$ (SOI). The effective Dresselhaus field is described by

$$
\mathbf{B}^{\mathrm{SO}}(\mathbf{k})=\frac{2 \beta}{g \mu_{\mathrm{B}}}\left(\begin{array}{l}
k_{y} \\
k_{x} \\
0
\end{array}\right)
$$

where we used a coordinate system with base vectors $\hat{x} \|$ [110], $\hat{y} \|[110]$ and $\hat{z} \|[001]$. Here, $g$ is the $g$ factor of the electrons, $\mu_{\mathrm{B}}$ is the Bohr magneton and $\beta$ is a constant proportional to the strength of the Dresselhaus SOI. The vectors determined by equation (1) in k-space are plotted in Fig. 1a.
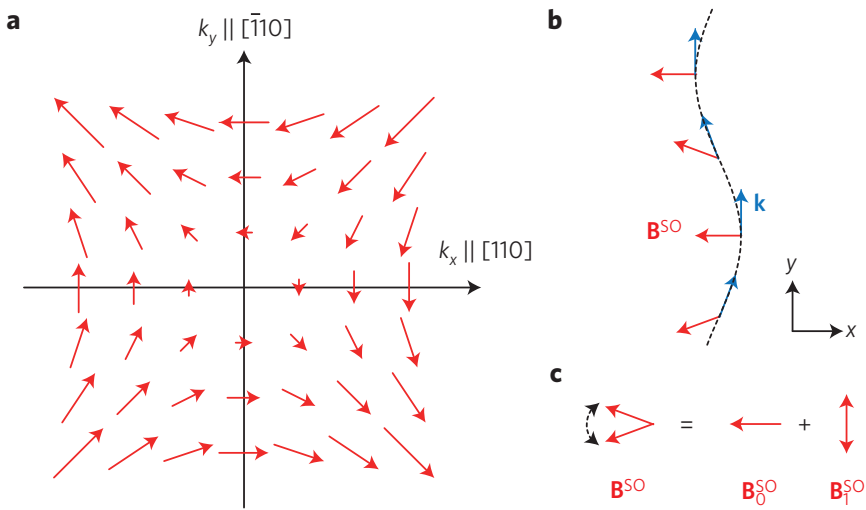

Figure 1 | Direction of momentum vectors and spin-orbit effective magnetic fields. a, Dresselhaus spin-orbit effective fields for different $\mathbf{k}$-vectors. $\mathbf{b}, \mathbf{k}$-vectors and Dresselhaus fields for electrons moving along a sinusoidal channel. $\mathbf{c}$, The travelling electrons experience static $\left(\mathbf{B}_{0}^{\mathrm{SO}}\right)$ and oscillating ( $\mathbf{B}_{1}^{\mathrm{SO}}$ ) fields that are normal to each other.

Now we consider electrons travelling along the sinusoidal channel shown in Fig. 1b. At each position of the channel, the deflection angle of the path determines the direction of the $\mathbf{k}$-vector. As a result, the moving electrons experience an effective magnetic field that swings with the frequency $f=v_{y} / \lambda$, where $v_{y}$ is the time-averaged $y$-component of the electron velocity and $\lambda$ is the period of the winding channel. In the reference frame moving with the electrons, the time-dependent effective magnetic field can be expressed as the sum of a static field $\left(\mathbf{B}_{0}^{\mathrm{SO}}\right)$ and an oscillating field $\left(\mathbf{B}_{1}^{\mathrm{SO}}\right.$; Fig. 1c). As $\mathbf{B}_{0}^{\mathrm{SO}}$ and $\mathbf{B}_{1}^{\mathrm{SO}}$ are normal to each other, they can drive coherent Rabi rotations when $h f$ matches the spin-splitting energy, that is, $h f=g \mu_{\mathrm{B}}\left|\mathbf{B}_{0}^{\mathrm{SO}}\right|$, where $h$ is the Planck constant. Note that a similar mechanism with different vector configurations also applies to electron systems with the Rashba SOI (ref. 20), or with both Dresselhaus and Rashba contributions.

To control the trajectory of travelling electrons, we adopted acoustically induced moving dots ${ }^{21,22}$ produced in an undoped 20-nm-thick GaAs/AlGaAs (001) quantum well, where the SOI acting on the two-dimensionally confined electrons is dominated by the Dresselhaus term ${ }^{10}$. The piezoelectric field induced by a surface acoustic wave (SAW) beam propagating along $\hat{y}(\|[\overline{1} 10])$ produces an array of potential wires, which align in the $\langle 110\rangle$ direction and move along $\hat{y}$ with the acoustic velocity $v_{\mathrm{SAW}} \approx 2.97 \mathrm{~km} \mathrm{~s}^{-1}$. A Ti film with $3-\mu \mathrm{m}$-wide slits deposited on the wafer partially screens the piezoelectric field and produces moving dots that travel along

${ }^{1}$ NTT Basic Research Laboratories, NTT Corporation, 3-1 Morinosato-Wakamiya, Atsugi, Kanagawa 243-0198, Japan, ${ }^{2}$ Department of Materials Science, Tohoku University, 6-6-02 Aramaki-Aza Aoba, Aoba-ku, Sendai 980-8579, Japan, ${ }^{3}$ Paul-Drude-Institut für Festkörperelektronik, Hausvogteiplatz 5-7, 10117 Berlin, Germany. *e-mail: sanada.haruki@lab.ntt.co.jp. 

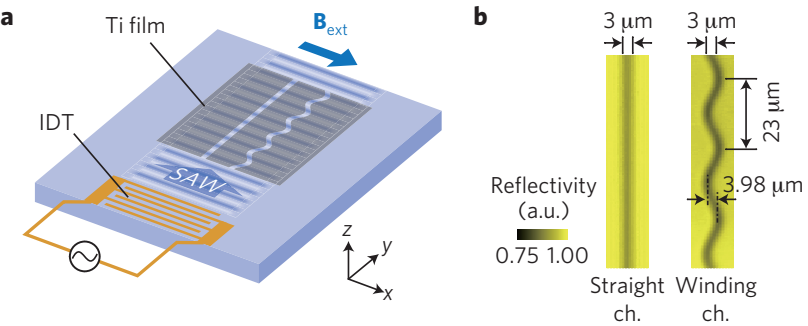

Figure 2 | Experimental set-up for mobile spin resonance. a, Schematic view of the sample. An interdigital transducer (IDT) formed on the quantum well wafer generates SAWs propagating along $\hat{y}$ (||[11ㅣ]). A Ti film with slits defines the straight and winding channels for the moving piezoelectric dots. We set the sample in an electromagnet so that $\mathbf{B}_{\text {ext }}$ is parallel to the $x$ axis. $\mathbf{b}$, Optical reflectivity images of the straight and winding channels.

the channels formed beneath the slits (Fig. 2a). Here, we discuss experimental results for acoustically induced spin transport along
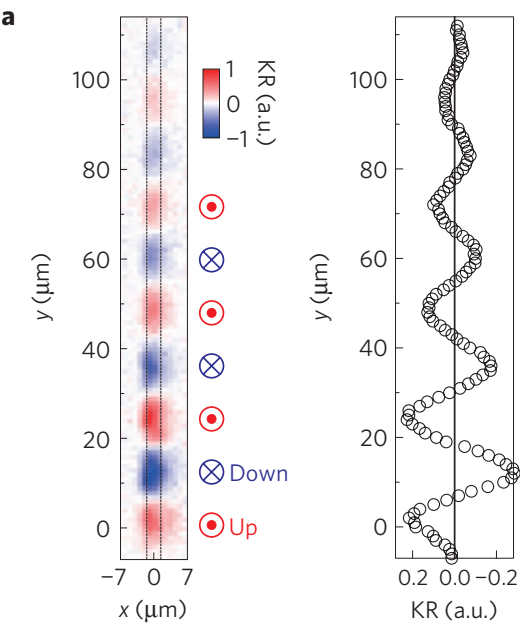

c
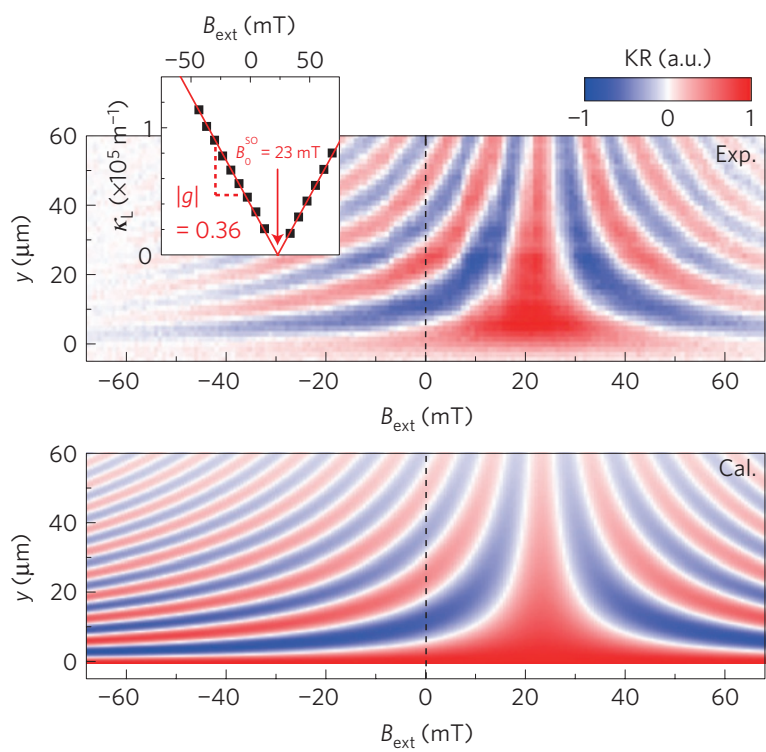

straight and winding channels whose dimensions are provided in the optical reflectivity images shown in Fig. 2 b.

We performed Kerr microscopy ${ }^{10,23}$ to measure the spin dynamics of the electrons moving along the channels. A circularly polarized pump light generates electron spins oriented along $\hat{z}$ at position $(x, y)=(0,0)$ on the channels, and a linearly polarized light probes the magneto-optic Kerr rotation, which is proportional to the spin density at the probe position. By scanning the probe position in the quantum well plane, we can obtain two-dimensional images of the spin distribution under a SAW.

Figure 3a maps the steady-state spin density for the straight channel in the absence of an external magnetic field. The long-lasting Kerr rotation signal indicates the successive carrier transport along the channel formed under the slit of the metal film. The Kerr rotation signal oscillations are attributed to the spin precession induced by $\mathbf{B}^{\mathrm{SO}}$ (ref. 9). Although the random fluctuations of $\mathbf{B}^{\text {SO }}$ due to the electron scattering in the moving dots induce the D'yakonov Perel' spin relaxation ${ }^{24}$, the spin coherence is maintained for a very long time $(\sim 20 \mathrm{~ns})$ because of the
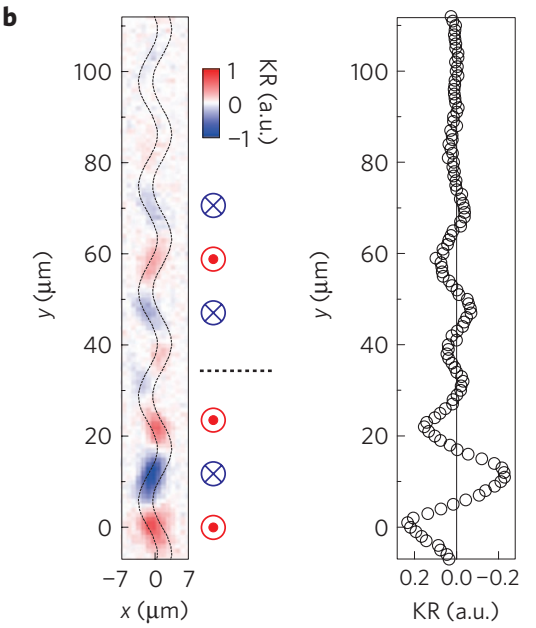

d
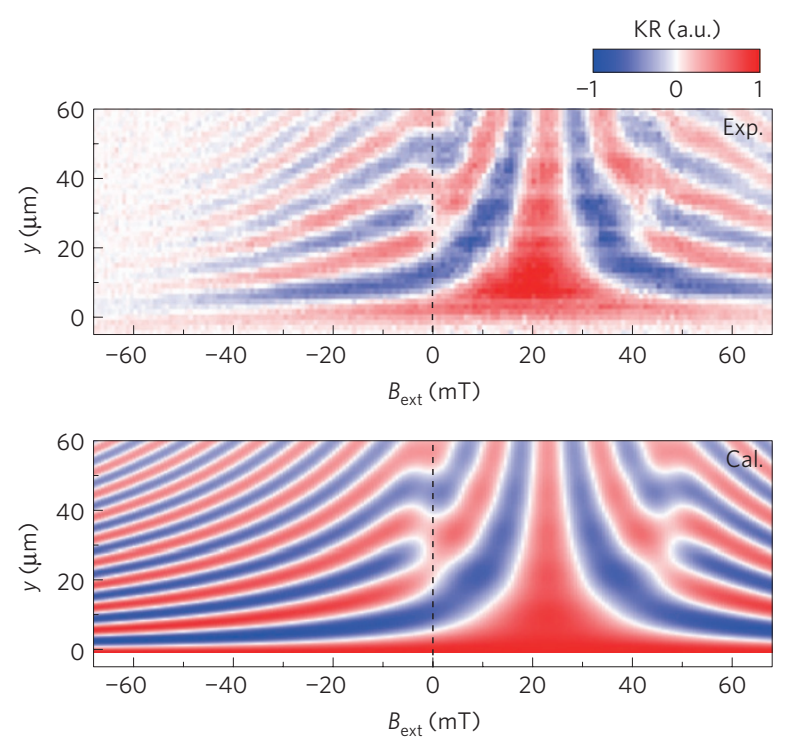

Figure $\mathbf{3}$ | Spin dynamics affected by $\mathbf{B}^{\text {SO }}$ and $\mathbf{B}_{\text {ext }}$. $\mathbf{a}, \mathbf{b}$, Kerr rotation $(K R)$ images of the straight (a) and winding (b) channels obtained by optically pumping spin-polarized electrons at $(x, y)=(0,0)$. The same data averaged along the $x$ axis are plotted as a function of $y$ on the right side of each Kerr rotation image. c,d, Comparison of experimental and calculated Kerr rotation signals plotted as a function of $B_{\text {ext }}$ and $y$ for the straight (c) and winding (d) channels. The inset in $\mathbf{c}$ shows the $B_{\text {ext }}$ dependence of the spatial Larmor frequency $\left(\kappa_{\mathrm{L}}\right)$ together with a fitted line from which we obtained the $\left|\mathbf{B}_{0}^{\mathrm{SO}}\right|$ and $g$-factor values described in the text. 
a

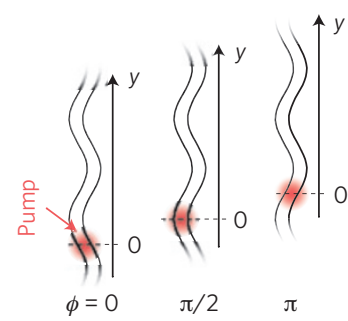

b

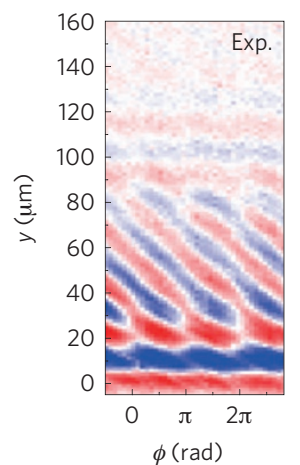

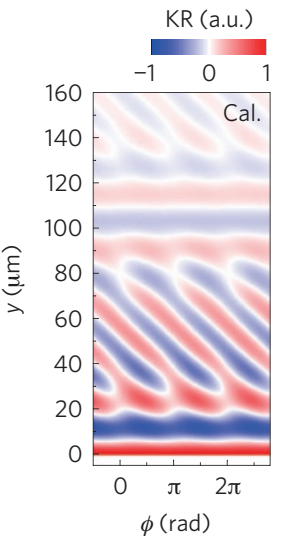

c
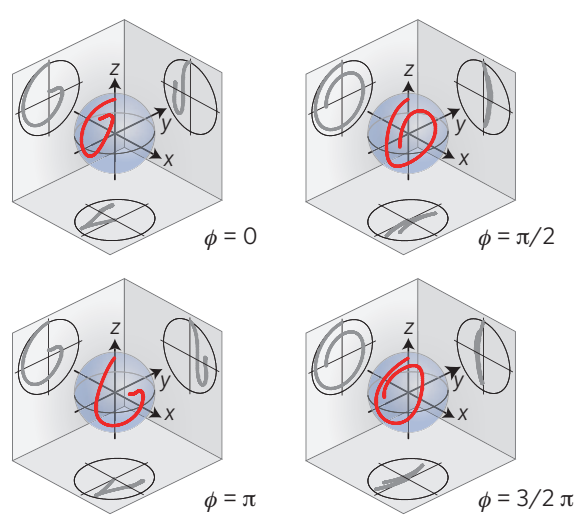

Figure 4 | Initial-phase dependence of the spin dynamics under resonant conditions $\left(\mathbf{B}_{\text {ext }}=\mathbf{0}\right)$. $\mathbf{a}$, The Kerr rotation measurements for the winding channel were carried out by pumping the spins at different positions, which define the initial phase $(\phi)$ of the alternating field $\mathbf{B}_{1}^{\text {SO }}$ as shown in the diagram. b. Comparison of experimental and calculated results for Kerr rotation (KR) plotted as a function of $\phi$ and $y$. c, Three-dimensional spin trajectories (red lines) calculated for the transport during $0 \leq y \leq 30 \mu \mathrm{m}$ plotted in Bloch spheres for $\phi=0, \pi / 2, \pi$ and $(3 / 2) \pi$. The grey lines are their projections on the three coordinate planes.

motional narrowing resulting from the SAW-induced mesoscopic confinement ${ }^{25}$. During this long spin lifetime, the electron spins with averaged wave vector $\overline{\mathbf{k}}=\left(m^{*} v_{\mathrm{SAW}} / \hbar\right) \hat{y}\left(m^{*}\right.$ is the effective mass of the electrons) behave as if they effectively experience only the average field given by

$$
\mathbf{B}_{0}^{\mathrm{SO}}=\frac{2 \beta m^{*} v_{\mathrm{SAW}}}{g \mu_{\mathrm{B}} \hbar} \hat{x},
$$

which is obtained by substituting $\overline{\mathbf{k}}$ into equation (1).

The application of $\mathbf{B}_{\text {ext }}$ along the $x$ axis enables us to obtain quantitative information about the spin dynamics during transport. The colour images in Fig. 3c show experimental and calculated Kerr rotation signals as a function of $y$ and $B_{\text {ext }}$ for the straight channel. When $B_{\text {ext }}$ approaches $23 \mathrm{mT}$, the precession slows almost to a halt, thus indicating that $\mathbf{B}^{\mathrm{SO}}$ is cancelled out at $B_{\text {ext }}=23 \mathrm{mT}$. In the inset of Fig. $3 c$, we fitted the $B_{\text {ext }}$ dependence of the Larmor frequency $\left(\kappa_{\mathrm{L}}\right)$ by:

$$
\kappa_{\mathrm{L}}=\frac{\mathrm{g} \mu_{\mathrm{B}}}{h v_{\mathrm{SAW}}}\left|\mathbf{B}_{\mathrm{ext}}+\mathbf{B}_{0}^{\mathrm{SO}}\right|
$$

from which we obtained $|g|=0.36$. In the following, we assume $g=-0.36$ in accordance with the negative $g$-value sign previously reported for similar quantum wells ${ }^{26}$. The calculated data shown in Fig. $3 c$ were obtained by assuming the above parameters $\left(B^{\mathrm{SO}}=23 \mathrm{mT}\right.$ and $\left.g=-0.36\right)$ and a spin decay length $L_{\mathrm{s}}=60 \mu \mathrm{m}$. The simulation well reproduces the experimental spin dynamics, thus showing that $\mathbf{B}^{\mathrm{SO}}$ acts on spins in the same way as the real magnetic field $\mathbf{B}_{\text {ext }}$ applied along the $x$ axis.

The effects induced by $\mathbf{B}_{1}^{\mathrm{SO}}$ appear in the spin dynamics of electrons travelling along the winding channel, which is designed to be close to the resonance condition $h v_{\mathrm{SAW}} / \lambda=g \mu_{\mathrm{B}}\left|\mathbf{B}_{0}^{\mathrm{SO}}\right|$. We set the origin (that is, the pump position) at a position where the dot velocity component along the $x$ axis takes its maximum value. As shown in Fig. $3 \mathrm{~b}$, the phase of the spin precession inverts when the probe position crosses $y \approx 30 \mu \mathrm{m}$. Such changes in precession dynamics were not observed for the straight channel (Fig. 3a), and appeared as peculiar features at around $B_{\text {ext }}=0$ and $46 \mathrm{mT}$ as shown in the experimental data in Fig. 3d. The fact that they appear symmetrically about $B_{\text {ext }}=23 \mathrm{mT}$ suggests that the corresponding phenomenon occurs when the total static field $\left|\mathbf{B}_{\text {ext }}+\mathbf{B}_{0}^{\mathrm{SO}}\right|=23 \mathrm{mT}$.
We simulated the spin dynamics under effective magnetic fields by solving a Bloch equation,

$$
\frac{\mathrm{d} \mathbf{S}}{\mathrm{d} t}=\frac{g \mu_{\mathrm{B}}}{\hbar}\left(\mathbf{B}_{\mathrm{ext}}+\mathbf{B}_{0}^{\mathrm{SO}}+\mathbf{B}_{1}^{\mathrm{SO}}\right) \times \mathbf{S}-\frac{v_{\mathrm{SAW}}}{L_{\mathrm{s}}} \mathbf{S}
$$

where we neglected equilibrium spin polarization and assumed that the longitudinal and transverse relaxation times were identical for simplicity. The parameters we used were $B_{0}^{\mathrm{SO}}=23 \mathrm{mT}, g=-0.36$, $B_{1}^{\mathrm{SO}}=10 \mathrm{mT}$ and $L_{\mathrm{s}}=60 \mu \mathrm{m}$. The experimental and calculated results in Fig. $3 \mathrm{~d}$ show excellent agreement and this proves the feasibility of the mobile spin resonance, which can be implemented even without external magnetic fields.

As the directions of the initial spins $\mathbf{S}_{0}(\| \hat{z}), \mathbf{B}_{0}^{\mathrm{SO}}(\| \hat{x})$ and $\mathbf{B}_{1}^{\mathrm{SO}}$ $(\| \hat{y})$ are orthogonal to each other, the spin dynamics under the resonance conditions strongly depends on the initial phase $(\phi)$ of $\mathbf{B}_{1}^{\mathrm{SO}}$, which is defined by the initial position of the spins along the winding channel as shown in Fig. 4 a. To confirm this, we measured Kerr rotation along the winding channel for different initial phases $(\phi)$ at $B_{\text {ext }}=0$. The experimental result in Fig. $4 \mathrm{~b}$ reveals a pattern that has periods of $\pi$ and $\sim 115 \mu \mathrm{m}$ along $\phi$ and $y$, respectively. Several peculiar features, which are also observed in Fig. 3d, indicate that $\mathbf{B}_{1}^{\text {SO }}$ drives the Rabi rotation effectively when $\phi=0$ and $\pi$, whereas it is ineffective when $\phi=\pi / 2$ and $(3 / 2) \pi$.

The simulation of the $\phi$-dependent Kerr rotation reveals the three-dimensional dynamics of the mobile spin resonance demonstrated here. The calculated pattern in Fig. $4 \mathrm{~b}$ was obtained by Bloch simulations using the parameters $B_{0}^{\mathrm{SO}}=23 \mathrm{mT}, g=-0.36$, $B_{1}^{\mathrm{SO}}=10 \mathrm{mT}$ and $L_{\mathrm{s}}=60 \mu \mathrm{m}$. The $\pi / 2$ pulse length of the ESR dynamics corresponds to $L_{\pi / 2}=29.5 \mu \mathrm{m}$, which determines the characteristic region of the pattern in Fig. $4 \mathrm{~b}$; the precession phase is almost unchanged for $0 \leq y \leq L_{\pi / 2}$ and $3 L_{\pi / 2} \leq y \leq 4 L_{\pi / 2}$, whereas it gradually shifts for $L_{\pi / 2} \leq y \leq 3 L_{\pi / 2}$. The Rabi oscillation period of $4 L_{\pi / 2}=118 \mu \mathrm{m}$ is slightly longer than the expected value of $93 \mu \mathrm{m}$, which was calculated from the shape of the winding channel assuming only the $k$-linear terms of the Dresselhaus SOI. This minor discrepancy is probably due to the contribution of Rashba-type SOIs, which are expected to suppress $\mathbf{B}_{1}^{\mathrm{SO}}$, or to the fact that the actual trajectories of the electrons do not follow the shape of the slit exactly.

The phase sensitivity of the mobile spin resonance indicates that we can prepare arbitrary spin states in the absence of external 
magnetic fields. Figure $4 \mathrm{c}$ shows the three-dimensional trajectories of the spin dynamics in the Bloch sphere calculated with the parameters defined above. When $\phi=\pi / 2$ and $(3 / 2) \pi$, the spin trajectories are always close to the $y-z$ plane. This motion is almost the same as the spin precession around the $x$ axis in the straight channel and allows spin preparation only in the $y-z$ plane. In contrast, when $\phi=0$ and $\pi$, the spin gradually acquires components along the $x$ axis. This fact indicates that the coherent Rabi rotation induced by the mobile spin resonance enables us to prepare any three-dimensional spin direction in the Bloch sphere by controlling the amplitude and transport length of the winding channel.

The experimental results presented here reveal coherent spin rotation using geometrically controlled SOI without any real magnetic fields. The phase-sensitive spin dynamics under resonance conditions suggests the applicability of general pulsed-ESR techniques such as the spin-echo sequence produced by connecting the winding and straight channels in series, although the effectiveness will depend on the decoherence mechanism of the target spin systems. The mobile spin resonance technique can also be extended to the electron spin transport induced by a lateral electric field ${ }^{17,27}$, if their trajectories can be well defined by nanofabrication or gateinduced confinement. Furthermore, combination with electricfield-dependent Rashba SOI (ref. 28) or with gate-defined moving quantum dots ${ }^{18}$ will enable the external control of the travelling spin coherence, thus opening the way for quantum gate operations on flying spin qubits in solid-state systems. Recent advances in related research, such as single electron transfer between static quantum $\operatorname{dots}^{22,29}$ or the spin lifetime extension using the persistent spin helix ${ }^{30}$, may open avenues for further development of the present technique towards quantum information applications.

\section{Methods}

The sample was a 20 -nm-thick undoped GaAs single quantum well with short-period GaAs/AlAs barriers (30\% average $\mathrm{Al}$ content) grown by molecular-beam epitaxy on a (001) semi-insulating GaAs substrate. The quantum well was located $485 \mathrm{~nm}$ below the surface. A 50-nm-thick Al film deposited on top of the sample was processed by electron-beam lithography into interdigital transducers, which were designed for operation at a SAW wavelength of $2.56 \mu \mathrm{m}$ and a frequency of $1.16 \mathrm{GHz}$. Rayleigh SAWs propagating along [110] with a SAW velocity $v_{\mathrm{SAW}} \sim 2.97 \mathrm{~km} \mathrm{~s}^{-1}$ produce the one-dimensional lateral confinement of the piezoelectric potential. In the SAW propagation area, we formed a Ti film with 3- $\mu \mathrm{m}$-wide slits with straight or sinusoidal centre lines as shown in Fig. 2b. The film partially screens the piezoelectric fields in areas other than the channel formed beneath the slits. The resultant potential produces moving dots that travel along the channel. The dot velocity component along $\hat{y}$ is determined by $\mathbf{v}_{\mathrm{SAW}}$.

The spin dynamics during transport was measured using spatially resolved Kerr rotation microscopy with a pair of Ti:sapphire lasers. Circularly polarized pump light (with an average power of $3.5 \mu \mathrm{W}$ ) was focused at a certain position on the channel, and the Kerr rotation angle of the reflected linearly polarized probe light $(1 \mu \mathrm{W})$ was measured with a balanced detection technique. The pump light was modulated between left- and right-circular polarizations at $50.1 \mathrm{kHz}$, and the probe light was chopped by using an acousto-optic modulator at $52.0 \mathrm{kHz}$. The difference frequency $(1.9 \mathrm{kHz})$ was used as a reference for lock-in detection. The full-width at half-maximum spot size of the normally incident probe beam was approximately $3 \mu \mathrm{m}$, whereas the waist size of the obliquely incident pump beam was $6 \mu \mathrm{m}$. All of the measurements were carried out at $8 \mathrm{~K}$.

Received 29 October 2012; accepted 6 February 2013; published online 17 March 2013

\section{References}

1. Loss, D. \& DiVincenzo, D. P. Quantum computation with quantum dots. Phys. Rev. A 57, 120-126 (1998).

2. De Sousa, R. \& Das Sarma, S. Electron spin coherence in semiconductors: Considerations for a spin-based solid-state quantum computer architecture. Phys. Rev. B 67, 033301 (2003).

3. Jelezko, F., Gaebel, T., Popa, I., Gruber, A. \& Wrachtrup, J. Observation of coherent oscillations in a single electron spin. Phys. Rev. Lett. 92, 076401 (2004).

4. Koppens, F. H. L. et al. Driven coherent oscillations of a single electron spin in a quantum dot. Nature 442, 766-771 (2006).

5. Hanson, R., Kouwenhoven, L. P., Petta, J. R., Tarucha, S. \& Vandersypen, L. M. K. Spins in few-electron quantum dots. Rev. Mod. Phys. 79, 1217-1265 (2007).
6. Bluhm, H. et al. Dephasing time of GaAs electron-spin qubits coupled to a nuclear bath exceeding 200 us. Nature Phys. 7, 109-113 (2010).

7. Winkler, R. Spin-Orbit Coupling Effects in Two-Dimensional Electron and Hole Systems (Springer Tracts in Modern Physics, Vol. 191, Springer, 2003).

8. Sogawa, T. et al. Transport and lifetime enhancement of photoexcited spins in GaAs by surface acoustic waves. Phys. Rev. Lett. 87, 276601 (2001).

9. Stotz, J. A. H., Hey, R., Santos, P. V. \& Ploog, K. H. Coherent spin transport through dynamic quantum dots. Nature Mater. 4, 585-588 (2005).

10. Sanada, H. et al. Acoustically induced spin-orbit interactions revealed by two-dimensional imaging of spin transport in GaAs. Phys. Rev. Lett. 106, 216602 (2011).

11. Kato, Y. et al. Gigahertz electron spin manipulation using voltage-controlled g-tensor modulation. Science 299, 1201-1204 (2003).

12. Tokura, Y., van der Wiel, W. G., Obata, T. \& Tarucha, S. Coherent single electron spin control in a slanting Zeeman field. Phys. Rev. Lett. 96, 047202 (2006).

13. Pioro-Ladrière, M. et al. Electrically driven single-electron spin resonance in a slanting Zeeman field. Nature Phys. 4, 776-779 (2008).

14. Nowack, K. C., Koppens, F. H. L., Nazarov, Yu. V. \& Vandersypen, L. M. K. Coherent control of a single electron spin with electric fields. Science 318, 1430-1433 (2007).

15. Meier, L., Salis, G., Shorubalko, I., Gini, E., Schön, S. \& Ensslin, K. Measurement of Rashba and Dresselhaus spin-orbit magnetic fields. Nature Phys. 3, 650-654 (2007).

16. Frolov, S. M., Lüscher, S., Yu, W., Ren, Y., Folk, J. A. \& Wegscheider, W. Ballistic spin resonance. Nature 458, 868-871 (2009).

17. Kato, Y., Myers, R. C., Gossard, A. C. \& Awschalom, D. D. Coherent spin manipulation without magnetic fields in strained semiconductors. Nature 427, 50-53 (2004).

18. Golovach, V. N., Borhani, M. \& Loss, D. Holonomic quantum computation with electron spins in quantum dots. Phys. Rev. A 81, 022315 (2010).

19. Dresselhaus, G. Spin-orbit coupling effects in zinc blende structures. Phys. Rev. 100, 580-586 (1955).

20. Bychkov, Y. A. \& Rashba, E. I. Oscillatory effects and the magnetic susceptibility of carriers in inversion layers. J. Phys. C 17, 6039-6045 (1984).

21. Biermann, K., Couto, O. D. D., Seidel, W., Hey, R. \& Santos, P. V. Electronic channels for acoustic transport in semiconductor heterostructures. Appl. Phys. Lett. 96, 162106 (2010).

22. Hermelin, $\mathrm{S}$ et al. Electrons surfing on a sound wave as a platform for quantum optics with flying electrons. Nature 477, 435-438 (2011).

23. Hernández-Mínguez, A., Biermann, K., Lazić, S., Hey, R. \& Santos, P. V. Kerr detection of acoustic spin transport in GaAs (110) quantum wells. Appl. Phys. Lett. 97, 242110 (2010).

24. D'yakonov, M. I. \& Perel', V. I. Spin relaxation of conduction electrons in noncentrosymmetric semiconductors. Sov. Phys. Solid State 13, 3023-3026 (1972).

25. Stotz, J. A. H., Hey, R., Santos, P. V. \& Ploog, K. H. Enhanced spin coherence via mesoscopic confinement during acoustically induced transport. New J. Phys. 10, 093013 (2008).

26. Snelling, M. J., Blackwood, E., McDonagh, C. J., Harley, R. T. \& Foxon, C. T. B. Exciton, heavy-hole, and electron $g$ factors in type-I GaAs $/ \mathrm{Al}_{x} \mathrm{Ga}_{1-x} \mathrm{As}$ quantum wells. Phys. Rev. B 45, 3922-3925 (1992).

27. Kikkawa, J. M. \& Awschalom, D. D. Lateral drag of spin coherence in gallium arsenide. Nature 397, 139-141 (1999).

28. Nitta, J., Akazaki, T., Takayanagi, H. \& Enoki, T. Gate control of spin-orbit interaction in an inverted $\operatorname{In}_{0.53} \mathrm{Ga}_{0.47} \mathrm{As} / \mathrm{In}_{0.52} \mathrm{Al}_{0.48}$ As heterostructure. Phys. Rev. Lett. 78, 1335-1338 (1997).

29. Yamamoto, M., Takada, S., Bäuerle, C., Watanabe, K., Wieck, A. D \& Tarucha, S. Electrical control of a solid-state flying qubit. Nature Nanotech. 7, 247-251 (2012).

30. Koralek, J. D. et al. Emergence of the persistent spin helix in semiconductor quantum wells. Nature 458, 610-613 (2009).

\section{Acknowledgements}

We thank Y. Tokura, H. Yamaguchi and T. Tawara for useful discussions. This work was supported by JSPS KAKENHI Grant Number 24686004 and 23310097.

\section{Author contributions}

H.S., Y.K., H.G. and T.S. designed the experiments; K.O. grew the sample; H.S. and Y.K. performed the measurements; M.K, J.N. and P.V.S provided theoretical support and conceptual advice; H.S. wrote the manuscript; and all of the authors discussed the results and commented on the manuscript at all stages.

\section{Additional information}

Reprints and permissions information is available online at www.nature.com/reprints. Correspondence and requests for materials should be addressed to H.S.

\section{Competing financial interests}

The authors declare no competing financial interests. 